\title{
Uso de pruebas psicológicas en Honduras por psicólogos
}

Daniel Matamoros Batson, Germán Moncada Godoy, Ivette C. Rivera ${ }^{1}$

\section{RESUMEN}

Se investigaron las formas en que los psicólogos en Honduras se capacitan y manejan el uso de las pruebas psicológicas en su actividad profesional con el fin de determinar el grado de dependencia que tienen de las capacitaciones adquiridas en su formación académica en pruebas específicas, comparado con su capacitación posterior extrauniversitaria.

Se utilizó una encuesta estructurada aplicada en dos ciudades de importancia, Tegucigalpa y San Pedro Sula, con una muestra de 150 psicólogos (88 \% mujeres y $12 \%$ hombres). Más de la mitad de los sujetos laboraban en el sector público. Elárea profesional de trabajo más reportada fue la clínica, seguida por la docencia y los recursos humanos.

Los sujetos informaron de la capacitación, manejo y uso profesional de 168 pruebas, principalmente de personalidad (26\%), inteligencia y desarrollo (15\%), exploración pedagógica (13\%) y aptitudes (7\%). Según esta investigación, las cinco pruebas más utilizadas son el test gestáltico visomotor de Bender, test de la familia, test casaárbol-persona (H-T-P), MMPI y el test de la figura humana, con cuatro de ellas gráficas (de dibujo).

Asimismo, las pruebas de inteligencia más utilizadas eran la de Raven, Weschler y Purdue. Se encontró que la preferencia por pruebas de dibujo se relaciona con su facilidad de aplicación con personas de poca escolaridad y su bajo costo. La muestra informó que $20 \%$ ( $N=33$ ) del total de las pruebas fueron aprendidas a profundidad en las universidades, un $79 \%$ del total $(\mathrm{N}=133)$ en forma autodidacta y un $66 \%$ del total $(N=111)$ en capacitaciones.

Al respecto, los sujetos señalaron la necesidad de capacitaciones sobre pruebas

\footnotetext{
${ }^{1}$ Profesores universitarios beneficiarios de una beca sustantiva de la DICYP. Escuela de Psicología, Facultad de Cienicas Sociales, UNAH: ivette.rivera@unah.edu.hn
} 
específicas, tener una actitud favorable hacia su uso, confiar en su validez y que sus decisiones de diagnóstico se basen en su juicio profesional, sin darle a las pruebas un peso preponderante. Resaltó, además, su preocupación por la falta de baremos nacionales. En consecuencia, se recomendó una mayor regulación por parte del Colegio de Psicólogos de Honduras.

Palabras clave: uso de pruebas psicológicas, formación para su uso, regulaciones, actitudes.

\section{ABSTRACT}

The present study explored which were the predominant test used by psychologists, with a sample of 150 psychologists in two major cities of Honduras. The main purpose was to determine whether the psychologists relied mainly on the test they were trained to use in university undergraduate courses or if they were using test trained for in different job settings or by individual self study methods.

A structured interview method was employed, and the sample consisted of 132 female and 18 male psychologists, mostly graduated from a five year university program (Licenciatura). More than $50 \%$ worked in the public sector, and the main professional areas were clinical psychology, university professor, and human resources.

The sample reported the training and use of a universe of 168 tests, including personality test (26\%), IQ and development scales (15\%), Achievement/Learning (13\%), and career development and planning (7\%). The study's results indicated that the five tests most used were the Bender Visual-Motor Gestalt Test, the Corman Family Drawing Test, the House-Tree-Person Test, MMPI, and the Draw a Person Technique, four of these being drawing techniques. The most popular Intelligence test were the RAVEN, the WESCHLER test, and the PURDUE NON-VERBAL TEST.

The results indicated that the popularity of the drawing test was related to their low cost and ease of use with subjects with lower academic skills. The subjects reported that training in the use of the 168 test was mostly self-directed (79\% of the test), followed by on-the-job training programs (66\%) and only $20 \%$ at undergraduate university programs.

The sample subjects indicated a need for training programs for different test. They also indicated a positive attitude towards test usage, a trust in their validity, and 
reported that in their diagnostic considerations they relied more in their professional training than in test results. A concern for a lack of national norms was noted. The subjects also emphasized the need for more regulatory measures by the Honduran Psychology Board.

Key words: psychological test usage, training, attitudes, need for regulations. 


\section{INTRODUCCIÓN}

Casi sesenta años después de la introducción de las pruebas psicológicas en Honduras, se consideró necesario investigar el rol que juegan en el desempeño profesional, elaborando un inventario de las mismas, su uso en las diferentes áreas profesionales y las formas en que se capacitan los psicólogos en este campo.

Asimismo, resultaba necesario averiguar cuál es la opinión que tienen los psicólogos sobre el uso de dichas pruebas, los problemas que enfrentan al utilizarlos en el contexto nacional y los factores que influyen positiva 0 negativamente en su uso. Es decir, que se justifica un estudio que brinde un panorama sobre el estado actual de sus usos, puesto que inciden en su aplicación la falta de controles adecuados para su adquisición, la relativa carencia de baremos hondureños (o su desconocimiento), los nuevos retos que enfrentan los psicólogos para su uso, incluyendo el acceso a las pruebas por internet, el uso de servicios computarizados para su administración, calificación e interpretación, el incremento significativo del número de pruebas disponibles (sin que exista un programa de capacitación para muchas pruebas nuevas en el mercado nacional), así como el riesgo de que los nuevos profesionales utilicen las mismas sin las orientaciones apropiadas.

Se pretende que este estudio permita a los psicólogos, a sus autoridades y a las universidades del país, contar eventualmente con un catálogo de las pruebas utilizadas y precisar en cuáles áreas y en cuáles pruebas se requiere de un mayor apoyo en su capacitación y posiblemente su adaptación o estandarización. En tal sentido, la presente investigación resulta ser una de las primeras realizadas en el país en este campo y se constituye en uno de los pocos estudios sobre la labor profesional de los psicólogos, con una muestra significativa de diferentes áreas y en dos ciudades importantes del país.

Para su realización se contó con el apoyo de la Dirección de Investigación Científica y Posgrado de la UNAH y la colaboración de un equipo profesional de encuestadores para la aplicación de la encuesta estructurada. No se encontraron dificultades significativas para la recolección de los datos y su análisis computarizado.

\section{Marco conceptual}

El entrenamiento en el manejo de las pruebas psicológicas constituye un componente importante de la formación académica y el desempeño profesional de 
la mayoría de los psicólogos en Honduras e incluye en la actualidad una formación en estadística descriptiva e inferencial, medidas de confiabilidad y de error, validez y significado de los resultados de las pruebas, así como una preparación a profundidad en un número no menor de treinta pruebas. Este énfasis se describe en dos trabajos relacionados con el desarrollo de la psicología en Honduras; el primero se encuentra en el libro Historia de la psicología en Honduras escrito por Víctor Donaire (2002), primer psicólogo hondureño graduado en Chile. Donaire relata cómo se promocionó la orientación vocacional y la psicopedagogía en diferentes instituciones educativas, en la década de los cincuentas, promoviendo el uso de diferentes pruebas psicológicas.

La otra fuente se refiere al uso de las pruebas en el área clínica en Honduras, la cual se fortaleció en los años sesenta desde la recién creada Carrera de Psicología de la Universidad Nacional Autónoma de Honduras. Desde sus inicios la falta de baremos nacionales fue un problema significativo del que tuvieron conciencia sus profesores.

La enseñanza y el uso de las pruebas psicológicas se expandieron en las siguientes décadas, por ejemplo, se identificaron 79 pruebas utilizadas en Honduras (con una muestra de 65 psicólogos en 35 centros laborales). A través de las tesis de grado de licenciatura de la Carrera de Psicología de la UNAH, se hizo un esfuerzo por elaborar normas regionales o nacionales, esfuerzo que se descontinuó a fines de la década de los setenta al eliminarse las tesis como requisito de graduación en dicha Universidad. Un ejemplo de un trabajo de normalización que todavía es utilizada en la actualidad es la tesis de María Elena Rivera, con el test Raven (Rivera, 1981).

En la actualidad se capacita a los alumnos de pregrado en diferentes universidades del país en la administración, calificación e interpretación de un amplio número de pruebas, a diferencia de muchos países en los cuales a nivel de pregrado solo se brinda una capacitación teórica sobre el uso de las pruebas psicológicas. En Honduras los psicólogos han expresado las siguientes preocupaciones sobre el uso de las pruebas:

1. El alto costo de las pruebas psicológicas, lo cual los convierte en inaccesibles u obligan a utilizar versiones ilegales de las mismas

2. La disponibilidad de muchas de las pruebas en Internet, en forma gratuita, reduciendo su confiabilidad.

3. La relativa falta de regulación en el uso de las pruebas por parte del Colegio de Psicólogos de Honduras, lo cual aseguraría que los usuarios tengan las 
calificaciones requeridas y que los psicólogos se capaciten adecuadamente en el uso de pruebas nuevas.

Ante la problemática antes expuesta, la presente investigación se orientó a revisar el panorama actual sobre el uso de las pruebas psicológicas en Honduras, con los siguientes objetivos:

1. Identificar las principales pruebas que los psicólogos manejan, precisando si su preparación ha sido a través de formación universitaria, en capacitaciones o en forma autodidacta.

2. Establecer las limitaciones que enfrentan en su uso, la proporción de tiempo que dedican a su uso y la percepción que tienen de su accesibilidad.

3. Determinar las actitudes de los psicólogos hacia el uso de las pruebas.

4. Establecer las principales prácticas que tienen los psicólogos con relación al uso de las pruebas y el nivel de conocimientos que poseen sobre las distintas competencias que se requieren para su uso.

\section{METODOLOGÍA}

La metodología fue descriptiva de tipo transversal con un enfoque cuantitativo.

1. Sujetos. La muestra se conformó con 150 psicólogos (sexo femenino, $\mathrm{N}=132$, sexo masculino, $\mathrm{N}=18$ ), todos laborantes en diferentes centros de trabajo. La edad promedio de los sujetos de la muestra fue de 37.3 años. Más de la mitad de los sujetos tenían de uno a diez años experiencia profesional y un $58 \%$ laboraban en el sector público. La principal área laboral fue la clínica (39 \%), seguido por la docencia universitaria (26.3\%) y los recursos humanos $(22.9 \%)$. Los porcentajes de las demás áreas son mucho menores: orientación vocacional $(12.5 \%)$, proyectos comunitarios $(5.0 \%)$, psicología jurídica 0 forense $(3.4 \%)$ y otros $(8.0 \%)$.

2. Instrumento. Se utilizó una encuesta desarrollada por los investigadores del presente estudio, tomando como referencia diferentes instrumentos utilizados en Colombia y España (ver González, 1977 y Muñiz y Fernández, 2000). El instrumento estaba conformado por 28 preguntas abiertas y cerradas, 
abarcando diferentes áreas, incluyendo el número y tipo de pruebas utilizadas, las características de las mismas, la valoración de la importancia de las pruebas en su desempeño profesional y los problemas para su uso. La encuesta no incluía una lista de pruebas. En la segunda parte del instrumento se pedía el nivel de acuerdo con diferentes afirmaciones sobre la importancia de las pruebas (totalmente de acuerdo, de acuerdo, en desacuerdo y totalmente en desacuerdo). El tiempo promedio para su aplicación fue de treinta minutos por persona.

3. Procedimiento. Se hizo una selección intencional de los sujetos buscando contar con profesionales de las diferentes áreas laborales (clínica, social, educativa e industrial). Se asistió a sus centros de trabajo informando el objetivo de la investigación y solicitando su colaboración. El equipo investigador aplicó un cincuenta por ciento de las encuestas y contrató a tres psicólogos para la aplicación del restante cincuenta por ciento. Treinta de las encuestas se aplicaron en la ciudad de San Pedro Sula y 138 en la ciudad de Tegucigalpa.

Después de una breve introducción por parte de los examinadores, la encuesta fue llenada por los sujetos en presencia de los encuestadores y se les informó que se manejarían los datos sobre su nombre y centro de trabajo en forma anónima. Se tabularon los datos en forma electrónica, excluyendo las respuestas de los sujetos que se referían a nombres genéricos de las pruebas y no a pruebas específicas (por ejemplo, test de dibujo en lugar de test de dibujo del árbol) o a nombres inexactos.

Seguidamente se realizó un análisis estadístico basado en análisis de frecuencias y porcentajes de acuerdo o desacuerdo con las afirmaciones presentadas. Se compararon las frecuencias reportadas con relación a diferentes variables, tales como el área profesional en que se desempeñaba, tipo de prueba y campo de aplicación, sector laboral y frecuencia de uso.

\section{RESULTADOS}

1. Pruebas más utilizadas

Los sujetos de la muestra identificaron un total de 168 pruebas utilizadas por los psicólogos en Honduras. Los datos de la tabla 1 indican las diez pruebas más 
populares. Los resultados señalan una preferencia por las pruebas proyectivas de personalidad, de tipo dibujo, realizadas por el propio sujeto (por ejemplo, test de la familia, test casa-árbol-persona, Machover) acompañadas por las pruebas de inteligencia y neuropsicológicas (específicamente la prueba Bender). El MMPI es la primera prueba objetiva reportada.

Tabla 1. Pruebas reportadas con mayor frecuencia por psicólogos en Honduras ( $N=150$ sujetos)

\begin{tabular}{|c|c|c|c|c|c|c|}
\hline $\begin{array}{c}\text { Nombre de la } \\
\text { prueba }\end{array}$ & $\begin{array}{c}\text { Porcen- } \\
\text { taje }\end{array}$ & Área & $\begin{array}{l}\text { Tipo de } \\
\text { prueba }\end{array}$ & Herramientas & $\begin{array}{c}\text { Edades } \\
\text { preferenciales }\end{array}$ & $\begin{array}{l}\text { Forma de } \\
\text { aplicación }\end{array}$ \\
\hline Test Bender & 36 & Neuropsicología & Proyectiva & Lápiz y papel & Niños/adultos & Administrado \\
\hline $\begin{array}{l}\text { Test de la } \\
\text { familia }\end{array}$ & 22 & Personalidad & Proyectiva & Lápiz y papel & Niños & Administrado \\
\hline $\begin{array}{l}\text { Test casa árbol } \\
\text { persona }\end{array}$ & 21.3 & Personalidad & Proyectiva & Lápiz y papel & Niños & Administrado \\
\hline MMPI & 20 & Personalidad & Objetiva & Estímulos & Adultos & $\begin{array}{l}\text { Autoadminis- } \\
\text { trado }\end{array}$ \\
\hline $\begin{array}{l}\text { Dibujo de la } \\
\text { figura humana, } \\
\text { de Machover }\end{array}$ & 18.7 & Personalidad & Proyectiva & Lápiz y papel & Niños/adultos & Administrado \\
\hline Test Raven & 15.3 & Inteligencia & Objetiva & Estímulos & Adultos & $\begin{array}{l}\text { Autoadminis- } \\
\text { trado }\end{array}$ \\
\hline WAIS/WISCI & 12.7 & Inteligencia & Objetiva & Estímulos & Niños/adultos & Administrado \\
\hline $\begin{array}{l}\text { WPPSI } \\
\text { Test Purdue }\end{array}$ & 11.3 & Inteligencia & Objetiva & Estímulos & Adultos & $\begin{array}{l}\text { Autoadminis- } \\
\text { trado }\end{array}$ \\
\hline $\begin{array}{l}\text { Test de colores } \\
\text { de Luscher }\end{array}$ & 10.0 & $\begin{array}{l}\text { Recursos } \\
\text { humanos }\end{array}$ & Proyectiva & Estímulos & Adultos & Administrado \\
\hline $16 \mathrm{PF}$ & 9.3 & Personalidad & Objetiva & Estímulos & Adultos & $\begin{array}{l}\text { Autoadminis- } \\
\text { trado }\end{array}$ \\
\hline
\end{tabular}

Fuente: elaboración propia.

Dos de las pruebas de inteligencia (Raven y Purdue) eran de autoaplicación y de lápiz y papel. Se concluyó que los costos de administración (estímulos requeridos) y el tiempo de aplicación eran un criterio fundamental para la selección de las pruebas.

La tabla 2 indica el número de pruebas que los psicólogos conocen o utilizan en su práctica profesional. 
Tabla 2. Número de pruebas conocidas por área profesional o de exploración

\begin{tabular}{|l|c|l|c|}
\hline \multicolumn{1}{|c|}{ Área de la prueba } & $\begin{array}{c}\text { Número } \\
\text { reportado }\end{array}$ & \multicolumn{1}{|c|}{ Pruebas principales } & $\begin{array}{c}\% \text { del } \\
\text { total }\end{array}$ \\
\hline Aptitudes & 12 & $\begin{array}{l}\text { ABI, DAT-5, TEA, TP, } \\
\text { CARAS, EFAI }\end{array}$ & 7.1 \\
\hline $\begin{array}{l}\text { Clínica/cuestionarios } \\
\text { Clínica/escalas }\end{array}$ & 4 & $\begin{array}{l}\text { CAEPO, STAIC, STAI, TQ } \\
\text { CMAS-R, EAE, ASPA, }\end{array}$ & 2.3 \\
\hline Clínica/otros & 12 & $\begin{array}{l}\text { BECK, ZUNG, CRS-R } \\
\text { Test Connors, FAD, } \\
\text { Achenbach, CAMDEX }\end{array}$ & 7.1 \\
\hline $\begin{array}{l}\text { Inteligencia y } \\
\text { desarrollo } \\
\text { Intereses/valores }\end{array}$ & 26 & $\begin{array}{l}\text { WAIS, Purdue, Raven, } \\
\text { Barsit, Otis, TONI-II }\end{array}$ & 15.5 \\
\hline Neuropsicología & 15 & $\begin{array}{l}\text { Kuder, CIPSA, AVL, SPV, } \\
\text { Geist, Lee Thorpe }\end{array}$ & 4.7 \\
\hline Cognistat, CUMANIN, \\
$\begin{array}{l}\text { Bender del reloj, Luria } \\
\text { Pedagógico }\end{array}$ & 22 & $\begin{array}{l}\text { FACILITO, TALE, BASC, } \\
\text { TIMMS, WEPMAN }\end{array}$ & 8.9 \\
\hline Personalidad & 13 & $\begin{array}{l}\text { MMPI, TAT, EPPS, EFY, } \\
\text { HTP, Machover, IRT }\end{array}$ & 25.1 \\
\hline Recursos humanos & 13 & $\begin{array}{l}\text { BTA-R, COMPETEA, } \\
\text { NEGO, TOM, DISC, IPV }\end{array}$ & 7.7 \\
\hline
\end{tabular}

$\mathrm{N}$ total $=168$ pruebas, no se incluyen las pruebas que fueron señaladas en forma genérica.

El total refleja el total de las pruebas identificadas por la muestra, en el cual se destaca la predominancia de las pruebas de personalidad ( $25.9 \%$ del total). Las pruebas de inteligencia constituyen la segunda área de importancia (15.5\%) seguido por las pruebas del área pedagógica ( $13.1 \%$ del total). Se señala el informe de otras pruebas neuropsicológicas además del Bender, tal como la prueba Cognistat y las pruebas de Luria, las cuales son muy especializadas (8.9\% del total). En general, se mantiene la proporción del tipo de pruebas que son enseñadas en las universidades y refleja la demanda de evaluaciones de personalidad e inteligencia como la práctica tradicional de los psicólogos.

El gráfico 1 refleja la proporción de pruebas conocidas por área, agrupando las pruebas de la práctica clínica en una sola área. Las pruebas de personalidad son las predominantes (26\%) seguidas por las de inteligencia y desarrollo (15\%), lo que refleja la formación recibida en las universidades. A pesar de los esfuerzos que se realizan en la empresa privada para capacitar a los psicólogos sobre el uso de nuevas pruebas, su aporte al total resulta menor, indicando un desconocimiento de muchos psicólogos de las pruebas utilizadas en este campo. 
Gráfico 1. Distribución de pruebas conocidas, por área

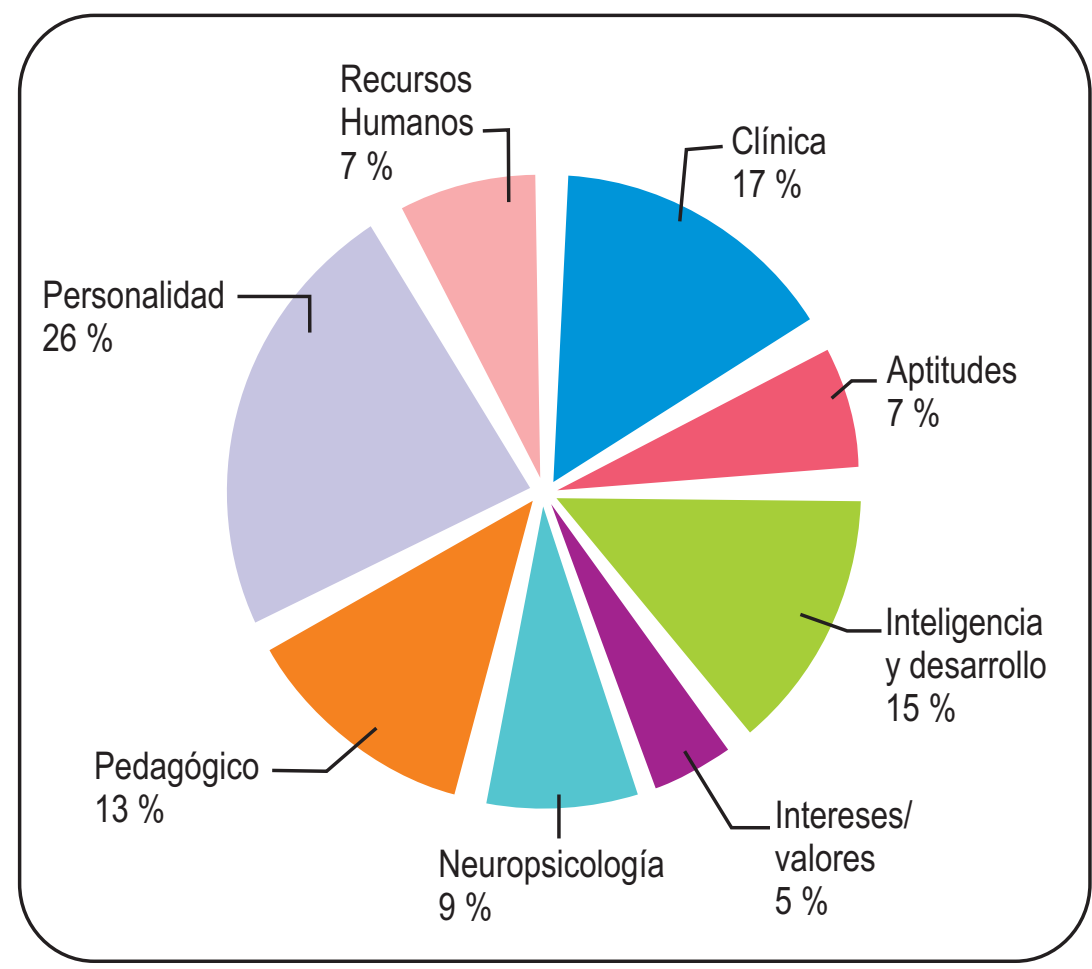

2. Principales pruebas utilizadas por área de desempeño profesional

Los sujetos informaron cuáles eran las pruebas más utilizadas de acuerdo a su área profesional. Los resultados se demuestran en la tabla 3.

Tabla 3. Pruebas más reportadas, por área

\begin{tabular}{|c|c|c|c|c|c|c|}
\hline $\begin{array}{l}\text { Prueba más } \\
\text { reportada }\end{array}$ & Docencia & $\begin{array}{l}\text { Recursos } \\
\text { humanos }\end{array}$ & Clínica & $\begin{array}{c}\text { Proyectos } \\
\text { comunitarios }\end{array}$ & $\begin{array}{l}\text { Orientación } \\
\text { vocacional }\end{array}$ & $\begin{array}{l}\text { Psicología } \\
\text { jurídica }\end{array}$ \\
\hline 1 & Bender & MOSS & Bender & Bender & Raven & MMPI-168 \\
\hline 2 & DISC & Raven & $\begin{array}{l}\text { Test de la } \\
\text { familia }\end{array}$ & HTP & Lee Thorpe & $\begin{array}{l}\text { Test de la } \\
\text { familia }\end{array}$ \\
\hline 3 & WPPSI & Purdue & MMPI & MMPI & Purdue & HTP \\
\hline 4 & $\begin{array}{l}\text { Test de la } \\
\text { familia }\end{array}$ & 16PF & HTP & WISC & DAT & Raven \\
\hline 5 & DFH & DISC & WISC-R & McMaster & HMP & DFH \\
\hline 6 & OTIS & Barsit & DFH & Goodenough & Holland & Beck \\
\hline 7 & HTP & Cleaver & Raven & Rorschach & Kuder & $\begin{array}{l}\text { Test de } \\
\text { experiencias } \\
\text { traumáticas }\end{array}$ \\
\hline
\end{tabular}




\begin{tabular}{|l|l|l|l|l|l|l|}
\hline $\begin{array}{c}\text { Prueba más } \\
\text { reportada }\end{array}$ & Docencia & $\begin{array}{c}\text { Recursos } \\
\text { humanos }\end{array}$ & \multicolumn{1}{|c|}{ Clínica } & $\begin{array}{c}\text { Proyectos } \\
\text { comunitarios }\end{array}$ & $\begin{array}{c}\text { Orientación } \\
\text { vocacional }\end{array}$ & $\begin{array}{c}\text { Psicología } \\
\text { jurídica }\end{array}$ \\
\hline 8 & WISC-R & CPI & WPPSI & $* * * *$ & MMPI & $\begin{array}{l}\text { Bender } \\
\text { Koppitz }\end{array}$ \\
\hline 9 & IPG & HMP & Luscher & $* * * *$ & $* * * *$ & Mini-Mult \\
\hline 10 & MBTI & Mini Mult & WAIS & $* * * *$ & $* * * *$ & \\
\hline & $\mathrm{N}=56$ & $\mathrm{~N}=29$ & $\mathrm{~N}=57$ & $\mathrm{~N}=8$ & $\mathrm{~N}=9$ & $\mathrm{~N}=9$ \\
\hline
\end{tabular}

Fuente: elaboración propia.

Las árıas de docencia y clínica tienen núrneros repor ados muy sirnilares ( $\mathrm{N}=56$ y $N=57$, respectivamente), pero son las más mencionadas; les siguen el área de recursos humanos con un número menor $(\mathrm{N}=39)$. Sin embargo, en las áreas de proyectos comunitarios, orientación vocacional y psicología jurídica el número es menor ( $N=8,9$ y 8 , respectivamente), indicando un menor énfasis en el uso de las pruebas.

3. Comparación de pruebas aprendidas en la universidad, en forma autodidacta 0 en capacitaciones

Se reportaron un total de 33 pruebas aprendidas en las universidades, en comparación a las 133 adquiridas en forma autodidacta. Esta última se orientó más al área de la personalidad ( $29 \%$ ), seguida por las pruebas de inteligencia (12\%). Las áreas de clínica y de recursos humanos predominan sobre las demás. Se denota un incremento importante en el número de pruebas del área de la neuropsicología, con once pruebas aprendidas en forma autodidacta. Incluyendo el Cunanin, el test de dominancia cerebral de Harris, la figura de Rey y el test del dibujo del reloj.

Los números menores corresponden a las áreas de proyectos comunitarios y de orientación vocacional. El test de los colores de Lüscher aparece como la prueba con más demanda (18\%), seguido por el test de persona bajo la lluvia $(10 \%)$.

Un total de 111 pruebas fueron reportadas como aprendidas en capacitaciones. Los profesionales del área de orientación vocacional informaron haber recibido más capacitaciones (2.5 por persona, en promedio), seguido por recursos humanos (1.6 por persona). Los profesionales del sector privado informan haber recibido más capacitaciones (3.5 por persona) que aquellos del sector público (1.9 por persona). 
4. Pruebas disponibles en Honduras

Los sujetos reportaron que, aproximadamente, en Honduras hay disponibilidad de 142 diferentes pruebas psicológicas. Las primeras diez pruebas que por lo menos diez psicólogos informaron como disponibles se presentan en la tabla 4 .

Tabla 4. Pruebas disponibles en Honduras para los psicólogos

\begin{tabular}{|l|c|c|l|}
\hline \multicolumn{1}{|c|}{ Prueba } & $\begin{array}{c}\text { Número de } \\
\text { psicólogos que lo } \\
\text { reportaron como } \\
\text { disponible }\end{array}$ & $\begin{array}{c}\text { Porcentaje de } \\
\text { psicólogos que } \\
\text { lo reportaron } \\
\text { como disponible }\end{array}$ & \multicolumn{1}{|c|}{$\begin{array}{c}\text { Tipo de } \\
\text { prueba }\end{array}$} \\
\hline Bender & 76 & 50.6 & Neuropsicología \\
\hline MMPI & 59 & $39-3$ & Personalidad \\
\hline HTP & 55 & 36.7 & Personalidad \\
\hline Raven & 55 & 37.7 & Inteligencia \\
\hline Test de la familia & 43 & 28.7 & Personalidad \\
\hline 16 PF & 39 & 26.0 & Personalidad \\
\hline DFH & 34 & 22.7 & Personalidad \\
\hline WISC-R & 34 & 22.7 & Inteligencia \\
\hline Purdue & 20 & 20.0 & Inteligencia \\
\hline WAIS & 25 & 17.3 & Inteligencia \\
\hline
\end{tabular}

Fuente: elaboración propia.

Los datos indican que las pruebas disponibles reportadas por los psicólogos son principalmente aquellas que se han aprendido en las universidades. El test Bender se presenta nuevamente como la prueba más reportada por los sujetos. Se concluye que el uso de pruebas psicológicas en el desempeño profesional se mantiene dentro de los parámetros brindados por las universidades.

La disponibilidad de las pruebas se refiere a la posesión personal, en los centros de trabajo 0 en la práctica privada. Los resultados (ver tabla 5) indicaron que no hubo una diferencia significativa en el número de pruebas disponibles (7.62 pruebas para el psicólogo en el sector público y 7.87 pruebas en el sector privado). En el sector público se reportaron un total de 125 pruebas disponibles, en comparación con 107 en el sector privado. 
Tabla 5. Pruebas disponibles para los psicólogos por área y por sector

\begin{tabular}{|l|c|c|}
\hline \multicolumn{1}{|c|}{ Área } & Público & Privado \\
\hline Neuropsicología & 1 & 4 \\
\hline Inteligencia & 5 & 2 \\
\hline Personalidad & 8 & 3 \\
\hline $\begin{array}{l}\text { Pedagógico (inte- } \\
\text { reses, aptitudes) }\end{array}$ & 5 & 5 \\
\hline Recursos humanos & 1 & 5 \\
\hline Clínica & 0 & 1 \\
\hline & $\begin{array}{l}\mathrm{N}=20 \text { más } \\
\text { reportados }\end{array}$ & $\begin{array}{l}\mathrm{N}=20 \text { más } \\
\text { reportados }\end{array}$ \\
\hline
\end{tabular}

Fuente: elaboración propia.

Como era de esperarse, un número mayor de pruebas en el área de recursos humanos están disponibles en el sector privado. El tipo de prueba que se reportó como más disponible era el de personalidad, con un total de 35 pruebas, seguida por pruebas de inteligencia y desarrollo $(N=25)$.

El reporte sobre las pruebas que se aplican en promedio en forma mensual (ver tabla 6) confirma el patrón observado en los cuadros anteriores. Las pruebas de dibujo son las primeras cuatro reportadas como las más administradas mensualmente, seguidas por seis pruebas de estímulo enseñadas en las universidades.

Con relación al tipo de prueba que el psicólogo aplica, los resultados de la encuesta indicaron que un $82.3 \%$ de los sujetos estuvo de acuerdo con la afirmación de que siempre se deben administrar pruebas de personalidad y un $76.6 \%$ estuvo de acuerdo en considerar que siempre se deben aplicar pruebas de inteligencia.

Tabla 6. Comparación de resultados de diferentes tablas, según orden reportado

\begin{tabular}{|c|c|c|c|c|c|}
\hline Prueba & $\begin{array}{c}\text { Más } \\
\text { utilizada }\end{array}$ & $\begin{array}{l}\text { Aprendidoen } \\
\text { el pregrado }\end{array}$ & $\begin{array}{c}\text { Más } \\
\text { disponible }\end{array}$ & $\begin{array}{l}\text { Más aplicado } \\
\text { en promedio }\end{array}$ & $\begin{array}{l}\text { Promedio de } \\
\text { orden reportado }\end{array}$ \\
\hline Bender & 1 & 2 & 1 & 1 & 1.2 \\
\hline $\begin{array}{l}\text { Test de la } \\
\text { familia }\end{array}$ & 2 & 8 & 5 & 3 & 4.5 \\
\hline HTP & 3 & 5 & 3 & 4 & 3.7 \\
\hline MMI & 4 & 1 & 2 & 6 & 3.2 \\
\hline DFH & 5 & 4 & 7 & 2 & 4.5 \\
\hline
\end{tabular}




\begin{tabular}{|c|c|c|c|c|c|}
\hline Prueba & $\begin{array}{c}\text { Más } \\
\text { utilizada }\end{array}$ & $\begin{array}{l}\text { Aprendidoen } \\
\text { el pregrado }\end{array}$ & $\begin{array}{c}\text { Más } \\
\text { disponible }\end{array}$ & $\begin{array}{l}\text { Más aplicado } \\
\text { en promedio }\end{array}$ & $\begin{array}{l}\text { Promedio de } \\
\text { orden reportado }\end{array}$ \\
\hline Raven & 6 & 3 & 4 & 5 & 4.5 \\
\hline $\begin{array}{l}\text { WAIS/WISCI } \\
\text { WPPSI }\end{array}$ & 7 & 7 & 8 & 7 & 7.2 \\
\hline Purdue & 8 & 6 & 9 & 13 & 9.0 \\
\hline $\begin{array}{l}\text { Colores de } \\
\text { Luscher }\end{array}$ & 9 & No & No & 11 & No aplica \\
\hline $16 \mathrm{PF}$ & 10 & 9 & 6 & 8 & 8.2 \\
\hline
\end{tabular}

Fuente: elaboración propia.

El análisis del cuadro 6 indica que la prueba de Bender es la que más aparece en los diferentes listados, seguida por el MMPI y HTP, con menores, pero significativos porcentajes sobre el test de la familia, DFH y Raven, todos con igual reporte. El test de colores de Lüscher se reporta como una de las más utilizadas, aunque no fuera aprendida en pregrado o reportado como muy disponible. Las opiniones de los informantes se presentan en la tabla 7.

Tabla 7. Primeras diez categorías de pruebas más utilizadas, según opinión de los sujetos de la muestra

\begin{tabular}{|l|c|c|}
\hline \multicolumn{1}{|c|}{ Área } & $\begin{array}{c}\text { Se debe aplicar } \\
\text { siempre, en por- } \\
\text { centajes }\end{array}$ & $\begin{array}{c}\text { Se debe aplicar casi } \\
\text { siempre o siempre, } \\
\text { en porcentajes }\end{array}$ \\
\hline Inteligencia & 76.6 & 98.7 \\
\hline Personalidad & 82.3 & 97.2 \\
\hline Pruebas proyectivas & 43.1 & 76.4 \\
\hline Aptitudes & 30.5 & 68.1 \\
\hline Pruebas clínicas & 23.5 & 59.4 \\
\hline específicas & & \\
\hline Habilidades & 32.6 & 57.4 \\
\hline Educativas & 25.4 & 55.7 \\
\hline Intereses & 23.9 & 53.9 \\
\hline No conductuales & 22.2 & 54.1 \\
\hline Habilidades sociales & 18.8 & 45.6 \\
\hline
\end{tabular}

Fuente: elaboración propia.

Los resultados coinciden con aquellos presentados anteriormente que destacan la prevalencia de las pruebas de inteligencia y de personalidad en el proceso de evaluación. Las pruebas proyectivas, en especial las de dibujo, resaltan como una de las principales herramientas de los psicólogos 
encuestados. Esto hace referencia a estudios anteriores, en otros países, que señalaron que las pruebas proyectivas son la principal herramienta en la evaluación de la personalidad (González, 1977). Un estudio más reciente (Cámara, Nathan y Puente, 2000) señala cuatro pruebas proyectivas entre las primeras diez pruebas utilizadas para evaluación en los Estados Unidos.

En la pregunta que se hizo a los encuestados sobre el tiempo que dedicaban al uso de pruebas en su trabajo profesional, más de la mitad informó que utiliza menos del $40 \%$ de su tiempo al uso de las pruebas, aunque un $20 \%$ afirma que sí dedica entre un 61 a $80 \%$ de su tiempo a su manejo. En consecuencia, es válido concluir que la aplicación de las pruebas es parte importante de la labor de los psicólogos.

El hecho de que más del $50 \%$ de los sujetos utilizan $40 \%$ o menos en el uso de las pruebas puede deberse, además, a la preferencia por aquellas pruebas que requieren de poco tiempo para su administración, calificación e interpretación.

5. Actitud de los psicólogos hacia el uso de las pruebas psicológicas

Una serie de preguntas de la encuesta exploraron las actitudes de los psicólogos hacia las pruebas. Las razones positivas para su uso, con su porcentaje de acuerdo, se presentan en la tabla 8.

Tabla 8. Actitud de los psicólogos hacia el uso de las pruebas psicológicas

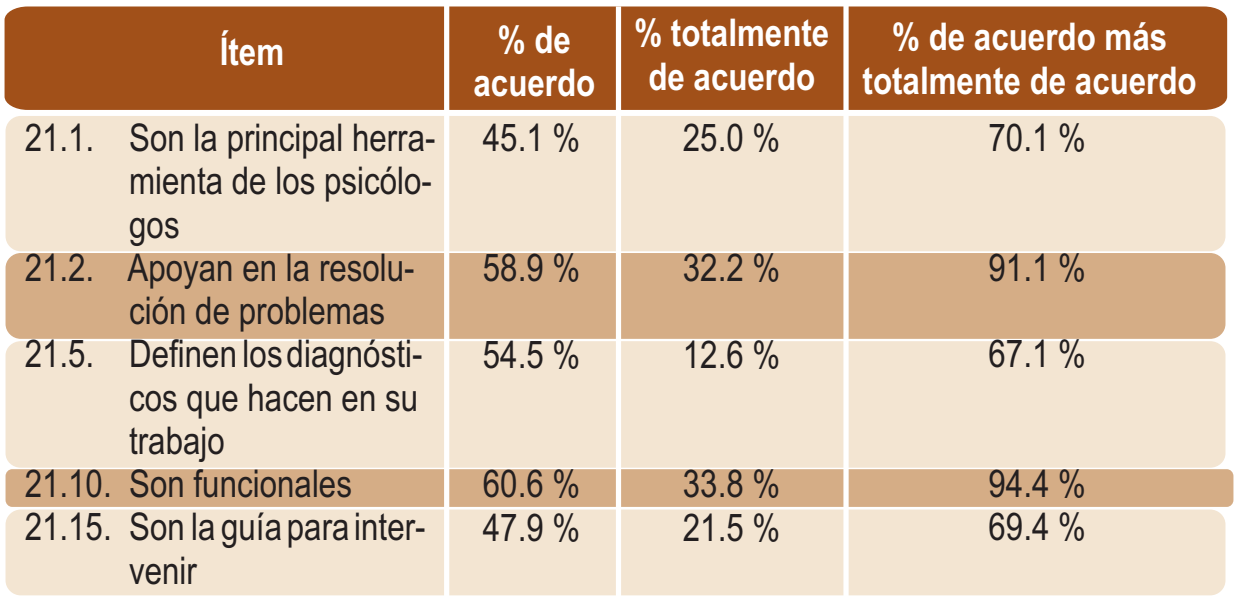

Fuente: elaboración propia. 
Los psicólogos reconocen la importancia de las pruebas psicológicas, pero los porcentajes que se refieren a un acuerdo total con las afirmaciones es bajo, lo que puede significar que existe una cierta reserva ante la afirmación de que hay una dependencia total hacia las mismas, por la convicción de que las demás habilidades personales y profesionales son más importantes que las pruebas. Con esto se reconoce que las pruebas son un apoyo, sin embargo, el criterio principal es la valoración clínica que realiza el psicólogo, basado en criterios amplios que incluyen la observación, la entrevista y el uso de las taxonomías diagnósticas $u$ otros criterios pertinentes. Las limitaciones $u$ objeciones relacionadas con el uso de las pruebas se presentan en la tabla 9.

Tabla 9. Opinión de los psicólogos sobre limitaciones u objeciones puestas al uso de las pruebas en Honduras

\begin{tabular}{|c|c|c|c|}
\hline Ítem & $\begin{array}{l}\% \text { de } \\
\text { acuerdo }\end{array}$ & $\begin{array}{l}\text { \% totalmente } \\
\text { de acuerdo }\end{array}$ & $\begin{array}{l}\% \text { de acuerdo más } \\
\text { totalmente de acuerdo }\end{array}$ \\
\hline $\begin{array}{l}\text { 21.16. Faltan barenos pro- } \\
\text { pios del contexto hon- } \\
\text { dureño }\end{array}$ & 28 & 66.0 & 94.0 \\
\hline $\begin{array}{l}\text { 21.3. Son instrumentos } \\
\text { costosos }\end{array}$ & 35.6 & 57.5 & 93.1 \\
\hline $\begin{array}{l}\text { 21.13. La preparación técni- } \\
\text { ca-científica en el uso } \\
\text { de los test es limitada }\end{array}$ & 47.5 & 25.5 & 73.0 \\
\hline $\begin{array}{l}\text { 21.7. Consideran muy poco } \\
\text { el contexto cultural de } \\
\text { la población hondure- } \\
\text { ña }\end{array}$ & 50.0 & 21.1 & 71.1 \\
\hline $\begin{array}{l}\text { 21.11. Suele hacerse un mal } \\
\text { uso de los test }\end{array}$ & 51.0 & 16.8 & 67.8 \\
\hline $\begin{array}{l}\text { 21.14. La confiabilidad de } \\
\text { los test en Honduras } \\
\text { es limitada }\end{array}$ & 52.8 & 14.1 & 66.9 \\
\hline $\begin{array}{l}\text { 21.12. El acceso a los test } \\
\text { es limitado }\end{array}$ & 43.2 & 20.5 & 63.7 \\
\hline $\begin{array}{l}21.6 \text { Tienen validez cues- } \\
\text { tionable }\end{array}$ & 41.3 & 5.6 & 46.9 \\
\hline
\end{tabular}

Fuente: elaboración propia.

La objeción principal es la falta de baremos apropiados para el contexto hondureño. La segunda se refiere a su costo y la falta de acceso a las mismas, lo cual justifica en parte el énfasis en las pruebas de lápiz y papel que no 
requieren de estímulos estructurados y que se pueden aplicar en un corto espacio de tiempo. Se objeta, además, la limitada preparación técnica-científica para su uso, lo cual es un aspecto importante por el gran número de pruebas que son aprendidos en forma autodidacta. La confiabilidad de las pruebas es cuestionada por la mayoría de los psicólogos, pero no su validez.

6. Manejo ético de las pruebas

Se le presentaron a los sujetos de la muestra una serie de planteamientos sobre los aspectos éticos del uso de las pruebas y los errores que pueden cometer los psicólogos. Los resultados se presentan en la tabla 10.

Tabla 10. Problemas éticos en el uso de las pruebas

\begin{tabular}{|c|c|c|c|}
\hline İtem & $\begin{array}{l}\% \text { nunca } 0 \\
\text { casi nunca }\end{array}$ & $\begin{array}{l}\% \text { algunas } \\
\text { veces }\end{array}$ & $\begin{array}{l}\% \text { casi siempre } \\
\text { o siempre }\end{array}$ \\
\hline 22.1. Errores al puntuar los test & 34.9 & 59.6 & 5.5 \\
\hline $\begin{array}{l}\text { 22.2. Errores al registrar los resul- } \\
\text { tados de las pruebas }\end{array}$ & 39.0 & 56.6 & 3.5 \\
\hline $\begin{array}{l}\text { 22.10. En ocasiones los psicólogos } \\
\text { acomodan los resultados de } \\
\text { acuerdo a diferentes intere- } \\
\text { ses }\end{array}$ & 61.3 & 34.0 & 4.2 \\
\hline $\begin{array}{l}\text { 22.7. Los examinados son debida- } \\
\text { mente informados del signifi- } \\
\text { cado de los resultados }\end{array}$ & 28.3 & 47.5 & 47.5 \\
\hline $\begin{array}{l}\text { 22.9. Se le da a los examinados } \\
\text { los detalles que solicitan }\end{array}$ & 12.7 & 26.8 & 60.6 \\
\hline $\begin{array}{l}\text { 22.3. Se etiqueta a las personas } \\
\text { basados en puntuaciones de } \\
\text { los test }\end{array}$ & 13.0 & 37.7 & 49.3 \\
\hline
\end{tabular}

Fuente: elaboración propia.

Los sujetos aceptaron que algunas veces ocurren en la puntuación (59.6\%), en el registro de los resultados ( $60 \%$ ) o por su falta de fidelidad hacia los resultados (34.0\%). Un porcentaje muy importante (61.3\%) defendió la integridad de sus evaluaciones planteando que nunca se acomodan los resultados a diferentes intereses.

De las primeras cinco pruebas más populares en Honduras, cuatro (test de dibujo) son calificadas con algún nivel de subjetividad, dependiendo de criterios específicos. Con relación a la exigencia ética de informar a los examinados 
sobre el significado de los resultados, solo un $47.5 \%$ de los psicólogos planteó que casi siempre o siempre se cumple con esta obligación. Esto puede deberse a la falta de atención individualizada a cada persona evaluada y a las políticas de las instituciones en que se laboran.

Se hizo una serie de preguntas sobre los factores externos que pueden afectar el uso de las pruebas y sus resultados, cuyos datos se muestran en la tabla 11.

Tabla 11. Factores externos que afectan el uso de las pruebas $(\mathrm{N}=168)$

\begin{tabular}{|l|c|c|c|}
\hline \multicolumn{1}{|c|}{ Ítem } & $\begin{array}{c}\% \text { nunca 0 } \\
\text { casi nunca }\end{array}$ & $\begin{array}{c}\text { \% algunas } \\
\text { veces }\end{array}$ & $\begin{array}{c}\text { \% casi siempre } \\
\text { o siempre }\end{array}$ \\
\hline 22.3. $\begin{array}{l}\text { Se etiqueta a personas basa- } \\
\text { do en puntuaciones de los } \\
\text { test }\end{array}$ & 13.0 & 37.7 & 49.2 \\
\hline 22.11. $\begin{array}{l}\text { Mucha de la información ob- } \\
\text { tenida a través de los test } \\
\text { resulta ser poco útil }\end{array}$ & 71.0 & 23.6 & 5.6 \\
\hline 22.12. & & & \\
\hline $\begin{array}{l}\text { Las predicciones basadas } \\
\text { en los test resultan muy } \\
\text { acertadas en el futuro }\end{array}$ & 3.4 & 19.2 & 77.4 \\
\hline
\end{tabular}

Fuente: elaboración propia.

Sobresale el uso de material fotocopiado (73.1\%) (casi siempre o siempre), lo que puede deberse al alto costo de las pruebas y sus manuales. El uso de material fotocopiado puede afectar de las siguientes maneras: 1) el uso de manuales 0 versiones anticuadas de las pruebas, al no mantenerse actualizado; 2) la poca calidad de las fotocopias, lo cual puede afectar el rendimiento del evaluado; 3 ) la pérdida de control sobre las pruebas, en los centros de reproducción.

Sobre la protección que se le debe dar a las pruebas, un $73 \%$ asegura que hay riesgo de que los materiales de las pruebas no estén guardados en lugares seguros. El requerimiento ético es que se tomen medidas para que los "test psicológicos no pierdan su valor por la divulgación al público" (Artículo 51, Código de Ética Profesional del Psicólogo, 1984).

Se exploró la percepción de los psicólogos sobre los controles que son necesarios para evitar el uso de las pruebas por personas de otras profesiones (por ejemplo, administradores de empresa). Al respecto, un $78.9 \%$ de los psicólogos consideraron que el uso de las pruebas debe restringirse a 
psicólogos calificados, mientras que un $72.4 \%$ consideró que el abuso y la utilización inapropiada de las pruebas es un problema serio en Honduras.

Con relación a esto, un 83.3 \% consideró que el Colegio de Psicólogos de Honduras debería ejercer un papel más activo para regular y mejorar el uso que se hace de las pruebas y un $82 \%$ estimó que se necesitan implementar normas legales para controlar los abusos más serios con tales pruebas.

Sobre la capacitación en el uso de las pruebas, más del $50 \%$ no estuvo de acuerdo con la afirmación de que los profesionales contaban con la información suficiente sobre la calidad de las pruebas disponibles a nivel nacional y un 74.3 $\%$ manifestó que la mejor forma de combatir el abuso y la utilización inapropiada era mediante el entrenamiento y la formación de los usuarios.

También se preguntó sobre el valor que las pruebas tienen en el proceso de evaluación. Los resultados se presentan en la tabla 12.

Tabla 12. Opinión de los psicólogos sobre el valor de las pruebas ( $\mathrm{N}=168)$

\begin{tabular}{|c|c|c|c|}
\hline Ítem & $\begin{array}{l}\% \text { nunca } 0 \\
\text { casi nunca }\end{array}$ & $\begin{array}{l}\% \text { algunas } \\
\text { veces }\end{array}$ & $\begin{array}{l}\% \text { casi siempre } \\
\text { o siempre }\end{array}$ \\
\hline $\begin{array}{l}\text { 22.4. Mantener en un lugar segu- } \\
\text { ro los materiales de los test }\end{array}$ & 11.1 & 15.9 & 73.1 \\
\hline $\begin{array}{l}\text { 22.6. Los lugares de aplicación no } \\
\text { siempre poseen las condi- } \\
\text { ciones que permitan a los } \\
\text { examinados un rendimiento } \\
\text { óptimo }\end{array}$ & 19.3 & 46.9 & 33.8 \\
\hline 22.8. Uso de material fotocopiado & 4.2 & 22.8 & 73.1 \\
\hline $\begin{array}{l}\text { 22.5. Los examinados no siempre } \\
\text { siguen las instrucciones }\end{array}$ & 30.8 & 50.3 & 18.9 \\
\hline
\end{tabular}

Fuente: elaboración propia.

Los resultados indican un respaldo al uso de las pruebas, ya que un 77 \% indicó que las predicciones basadas en las mismas eran muy acertadas. Un $77 \%$ rechazó la afirmación de que las pruebas eran poco útiles. Los sujetos reconocieron que el uso de las pruebas llevaba a etiquetar a las personas (por ejemplo, el MMI o el Bender). Con relación a su propia preparación para el manejo y uso de los mismos, los psicólogos consultados indicaron altos niveles de conocimientos (por encima de $80 \%$ así lo indicaron) en diversas áreas, tales como las pruebas de intereses vocacionales ( $86.8 \%$ ), las pruebas proyectivas 
(85.6\%), la confiabilidad de las pruebas (84.3\%) y las escalas de actitudes/valores (82.4\%). Estos datos se reflejan en la tabla 13.

Tabla 13. Confianza de los psicólogos en su conocimiento de las pruebas $(\mathrm{N}=168)$

\begin{tabular}{|l|c|c|c|}
\hline \multicolumn{1}{|c|}{ Ítem } & $\begin{array}{c}\text { \% reportaron } \\
\text { conocimiento } \\
\text { medio }\end{array}$ & $\begin{array}{c}\text { \% reportaron } \\
\text { conocimiento } \\
\text { alto }\end{array}$ & $\begin{array}{c}\text { \% reportaron } \\
\text { conocimiento } \\
\text { medio o alto }\end{array}$ \\
\hline Pruebas de personalidad & 32.6 & 66.0 & 98.6 \\
\hline Administración de una prueba & 14.5 & 83.4 & 97.9 \\
\hline Ética en el uso de las pruebas & 15.9 & 81.4 & 97.3 \\
\hline Manejo de pruebas de inteligencia & 24.5 & 72.0 & 96.5 \\
\hline Normas en el uso de las pruebas & 27.3 & 69.2 & 96.5 \\
\hline Manejo de pruebas de habilidades & 31.5 & 60.8 & 92.3 \\
\hline mentales & & & \\
\hline Pruebas de intereses vocacionales & 42.4 & 44.4 & 86.8 \\
\hline Pruebas proyectivos & 36.3 & 49.3 & 85.6 \\
\hline Confiabilidad & 40.0 & 44.3 & 84.3 \\
\hline Escalas de actitudes y valores & 38.0 & 44.4 & 82.4 \\
\hline
\end{tabular}

Fuente: elaboración propia.

Los encuestados indicaron además tener bajos niveles de conocimientos sobre ciertas áreas, las que se presentan en la tabla 14.

Tabla 14. Limitaciones reconocidas por psicólogos sobre temas de psicometría $(\mathrm{N}=158)$

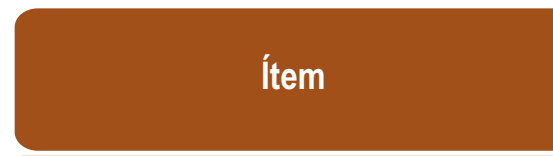

Elaboración de reactivos

Percentiles, deciles y cuartiles

Diseño de una prueba

Estandarización de pruebas

Pruebas neuropsicológicos

Análisis factorial

Correlación

Regresión linear

Fuente: elaboración propia.

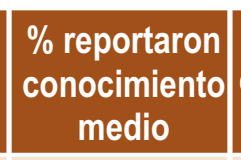

43.4

39.7

41.7

41.4

24.3

27.1

35.9

19.2

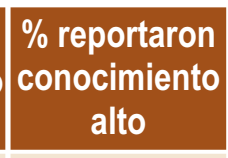

15.2

17.1

11.1

11.0

22.2

6.9

8.3

2.1

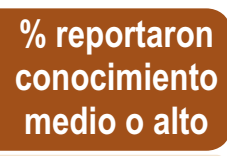

58.6

56.8

52.8

52.4

46.5

34.0

44.2

21.3

Un análisis de los resultados presentados en las tablas 13 y 14 indica un alto nivel de confianza en su manejo y el uso de las pruebas de inteligencia y 
habilidades mentales. Su dominio de las pruebas proyectivas y de escalas de actitudes/valores es ligeramente menor. Estos tipos de prueba son parte de los planes de estudio de las carreras universitarias.

No obstante, los psicólogos admiten una preparación muy limitada en temas importantes en análisis estadísticos avanzados y en pruebas neuropsicológicas. Esto puede ser un factor importante en la escasa producción de pruebas por los psicólogos en Honduras. Así es que el único ejemplo conocido de una prueba diseñada por psicólogos hondureños es la elaborada por Antonio Flores Arriaza, en tesis de grado en 1981.

\section{DISCUSIÓN}

El uso de las pruebas psicológicas no se limita principalmente a la formación universitaria, sino a sus necesidades laborales. Tienen una alta confianza en su uso y en la preparación que reciben en las universidades. Esta preparación es considerada como una base para adquirir conocimientos sobre muchas otras pruebas, ya sea en forma autodidacta o a través de capacitaciones, pasando de un promedio de 35 pruebas aprendidas en las universidades, hasta un promedio de 130 pruebas aprendidas en forma autodidacta y 110 por medio de capacitaciones.

El presente estudio señala el peligro de que se pueda perder el control en el buen manejo de estos instrumentos porque, en general, las orientaciones que se reciben en las universidades se mantienen, dándole prioridad a las pruebas del área clínica y de tipo proyectivo. Consecuentemente, resulta preocupante que para muchos psicólogos la prueba Bender es la única prueba neuropsicológica conocida.

No obstante lo antes señalado, existe un interés significativo por el uso de las pruebas en la mayoría de las áreas profesionales de la psicología, aunque su bajo uso en el área de proyectos comunitarios puede afectar la posibilidad de medir el impacto psicológico de los proyectos que se desarrollan en las comunidades a través de pruebas o escalas.

Los problemas reportados por los sujetos de la muestra durante esta investigación son los mismos que se indicaron desde hace décadas, especialmente la falta de baremos nacionales. Por tal razón, en el contexto nacional es determinante la preferencia de pruebas no verbales (de personalidad e inteligencia), aunque existe 
un gran interés en nuevas pruebas no verbales (por ejemplo, la prueba persona bajo la lluvia y el test de colores de Lüscher).

Al comparar las preocupaciones de los psicólogos hondureños con lo que revelan estudios de otros países, se evidencia que las preocupaciones sobre la falta de supervisión y control en el uso de las pruebas es compartido por investigadores de varios países. Para el caso, las políticas públicas adoptadas en Brasil, de tener un organismo estatal con la responsabilidad de aprobar el uso de pruebas específicas (Sistema para la Evaluación de Pruebas Psicológicas, SATEPSI; Primi, 2010), probablemente no sería apoyada por los psicólogos hondureños. Sin embargo, se enfatiza que debe existir un rol más preponderante para el Colegio de Psicólogos para este tema. Asimismo, las universidades deben tomar conciencia del papel decisivo que desempeñan en la selección de las pruebas que los psicólogos utilizarán en el futuro.

\section{CONCLUSIONES}

El presente estudio permite señalar cuáles son las pruebas que se están utilizando y en cuáles existen una mayor demanda de capacitación, lo cual puede ser llevado a cabo por diferentes instituciones. De esta manera, se concluye que las pruebas psicológicas más utilizadas por los psicólogos encuestados son el test gestáltico visomotor Bender, test de la familia, test árbol-casa-persona, MMPI y el test de la figura humana.

La mayoría de los encuestados indicaron que la base para el manejo de pruebas la obtuvieron durante la formación universitaria, permitiéndoles ser autodidactas en pruebas nuevas que aprenden en el transcurso de su vida laboral. En general, los profesionales confían en la validez de las pruebas psicológicas y optan por las económicas, por lo que las más usadas son las pruebas proyectivas gráficas. También se encontró como la limitante principal en el uso de las pruebas, la carencia de baremos nacionales.

En síntesis, se propone crear un catálogo de las pruebas psicológicas utilizadas en Honduras, con una ficha de cada prueba (con baremos nacionales, cuando sea posible); además, gestionar una reunión o una asamblea de psicólogos para discutir y tomar medidas sobre los nuevos retos que enfrentan en el uso de las pruebas psicológicas. 


\section{AGRADECIMIENTOS}

A la Dirección de Investigación Científica y de Posgrado de la UNAH, por su apoyo para la realización de esta investigación; igualmente, a los colegas que participaron como encuestadores y a los sujetos de la muestra por su colaboración.

\section{BIBLIOGRAFÍA}

Cámara, W.J.; Nathan, J.S. y Puente, A. (2000). Psychological Test Usage: Implications in Professional Psychology. Professional Psychology: Research and Practice, 31(2), 141-154.

Colegio de Psicólogos de Honduras. (1984). Ley Orgánica y Reglamentos del Colegio de Psicólogos de Honduras. Tegucigalpa: Editorial Escuela Superior del Profesorado.

Donaire, Victor M. (2002). Historia de la psicología en Honduras. Tegucigalpa: Universidad Nacional Autónoma de Honduras.

Flores Arriaza, J.A. (1981). El rendimiento académico de los alumnos de primer ingreso a la UNAH y su relación con el funcionamiento cognoscitivo. Tesis de licenciatura, UNAH, Tegucigalpa.

González, J. (1977). Test psicológicos en Colombia. Revista Latinoamericana de Psicología, 9(009), 429-435. Recuperado de: http://redalyc.uaemex.mx

Mejía, I. L. (1981). Validación del test de "dominós" en estudiantes de educación media de la capital de Honduras. Tesis de licenciatura, UNAH, Tegucigalpa.

Muñiz, J. y Fernández, J.R. (2000). La utilización de los test en España. Papeles del Psicólogo, 076, 41-49. Recuperado de: http://www.Redalyc.org

Primi, R. y Nunes, Carlos H.S. (2010). Psychological Testing \& Assessment in Brazil: Advances in Research and Federal Regulations. Testing International, 24, 4-6.

Rivera, M.E. (1981). Normalización del test de matrices progresivas Raven, escala general, en estudiantes de educación secundaria de la zona central de Honduras. Tesis de licenciatura, UNAH, Tegucigalpa. 